\title{
Introducción al dosier monográfico «Cambio climático, biodiversidad y ecología en el cómic»
}

\author{
Eduard Baile López \\ Universitat d'Alacant-Unicómic \\ Laura Caraballo \\ Université Clermont Auvergne \\ José Rovira-Collado \\ Universitat d'Alacant-Unicómic
}


Eduard Baile López es profesor contratado doctor en el Departament de Filologia Catalana de la Universitat d'Alacant. Forma parte de los grupos Recerca de Literatura Contemporània y Estudis Transversals: literatura i altres arts en les cultures mediterrànies. Su trayectoria docente se relaciona con asignaturas de normativa lingüística en la Facultad de Educación de su institución. Coordina, con José Rovira-Collado, el módulo El cómic en el aula de secundaria en el Máster Propio en Cómic y Educación de la Universitat de València. Su investigación se centra en el uso didáctico del cómic y en los estudios culturales. Ha coordinado varios monográficos como «Cómic y educación» en Tebeosfera (2019), también con Rovira-Collado. Es uno de los coordinadores académicos de las jornadas de Unicómic (desde 2011) y director del Aula de Cómic de la Universitat d'Alacant (desde 2017).

José Rovira-Collado es profesor contratado doctor en el área de Didáctica de la Lengua y la Literatura de la Universitat d'Alacant, de la que también es coordinador. Subdirector del Departamento de Innovación y Formación Didáctica en la Facultad de Educación. Especialista en literatura infantil y juvenil, la lectura multimodal y el uso de la tecnología para su enseñanza. Es coordinador académico de Unicómic desde 2007. Tiene más de cien publicaciones entre artículos académicos y capítulos de libros. También es administrador del blog http://literaturainfantilyjuvenileninternet.blogspot.com/

Laura Caraballo es historiadora del arte especializada en historieta latinoamericana, particularmente en la obra del dibujante Alberto Breccia y la historieta contemporánea argentina. Ha trabajado como docente, investigadora y curadora en Francia y Argentina. Actualmente enseña en la Universidad Bordeaux Montaigne, en la escuela de diseño ELISAVA Barcelona y en la Université Clermont Auvergne. En esta institución desarrolla un proyecto de investigación postdoctoral, bajo la dirección de la profesora Viviane Alary, sobre la función de la comunicación visual (específicamente, la historieta) en la reducción de la vulnerabilidad de poblaciones particularmente expuestas a riesgos y catástrofes naturales. 
El presente monográfico, que consiste en un completo dosier acerca del cómic como herramienta para analizar los efectos del cambio climático, así como instrumento concienciador en clave ecocrítica, es fruto de la colaboración entre el proyecto Investigation on Comics and Graphic Novels in the Iberian Cultural Area (acrónimo iCOn-MICs, COST Action 19119), gestionado a través del Centre de Recherches sur les Littératures et la Sociopoétique (CELIS) de la Université Clermont Auvergne, y el grupo Unicómic de la Universitat d'Alacant.

Por lo que respecta a iCOn-MICs, ${ }^{1}$ se trata del primer proyecto de su tipo en ámbito europeo, una empresa altamente ambiciosa que, bajo la dirección de Viviane Alary (Université Clermont Auvergne) en primera instancia y Jesús Jiménez-Varea (Universidad de Sevilla) en segundo lugar, tiene por objetivo la conformación de una comunidad de investigadores que, a su vez, permita construir un centro de recursos sobre el cómic ibérico. Más concretamente, este monográfico se enmarca en su Working Group 2, denominado "The Metamorphoses of Contemporary Iberian Comics» y cuyos responsables son Benoît Mitaine (University Paul Valéry - Montpellier 3), en calidad de Leader, y José Manuel Trabado (Universidad de León) como Scientific Advisor.

En cuanto al grupo Unicómic, ${ }^{2}$ responde a una asociación de carácter cultural vinculada a la Universitat d'Alacant y que es promotora de las jornadas de investigación académica sobre historieta más longevas en el ámbito universitario español. Su labor, enfocada especialmente a las aplicaciones pedagógicas del cómic, se refleja en las propias jornadas como eje central, pero también en cursos didácticos, redes docentes, el Aula de Cómic de la misma institución universitaria ${ }^{3}$ y, como colofón, la consagración en tanto que congreso internacional, ${ }^{4}$ de lo que se han derivado numerosas publicaciones especializadas.

En pleno siglo xxi, la reflexión sobre las catástrofes climáticas y la reducción de la biodiversidad del planeta a marchas forzadas se ha constituido como un tema de gran relevancia. La percepción progresiva de que nos acercamos a un punto de no retorno, si no se actúa de manera efectiva contra el deterioro del planeta y no se atienden las condiciones sanitarias y

\footnotetext{
${ }^{1}$ https://iconmics.hypotheses.org/

2 https://unicomic.blogspot.com/

${ }^{3}$ https://web.ua.es/es/aulacomic/

${ }^{4}$ Para la edición de 2018 ver: https://web.ua.es/es/unicomic/. Para la edición de 2021 ver: https://web.ua.es/ es/unicomic2021/
} 
de accesibilidad alimentaria de sus habitantes, mediante políticas socioeconómicas que hagan de contrapeso al capitalismo salvaje, inmerso ya en una fase de autofagocitamiento, ha dado pie a una línea de creación que pone el foco sobre el arte como recurso para analizar la realidad y, si cabe, denunciar los abusos neoliberales que la permeabilizan para plantear, así, alternativas sostenibles. Esto, que remite a las desigualdades torpemente escondidas entre los oropeles del consumismo y del despilfarro en los países privilegiados pero, quizá aún con más fuerza, a los abusos de Occidente hacia los territorios más desfavorecidos, of rece a los investigadores un corpus de obras en crecimiento y que ponen de manifiesto el poder de la cultura para revelar las miserias humanas y remover conciencias. En este sentido, el medio del cómic no ha permanecido ajeno y, por ello, los coordinadores de este monográfico hemos querido reunir un breve compendio de aportaciones que arrojen luz sobre el desarrollo de tal perspectiva ecocrítica, de raigambre progresista y carácter reivindicativo - en realidad, el medioambientalismo no se puede desligar de la perspectiva de género, de los queer studies, etc.- en diversidad de historietas, especialmente en el ámbito hispanoamericano aunque no exclusivamente.

El futuro, como se puede comprobar en los artículos seleccionados, ya está aquí y no es sino un paisaje herido de muerte, fotografía esencial del sistema económico dominante, un potente ente marginalizador que devora a sus hijos pero también a sí mismo. Ante este descenso inminente a los infiernos, el mundo de la historieta se revela como un ámbito propicio, merced a las virtudes de su lenguaje iconotextual para establecer espacios y temporalidades preñadas de valor metafórico — se destaca, por los analistas, la potencialidad del dibujo en la implementación de un distanciamiento crítico respecto a lo representado-, al análisis de las relaciones entre los grupos humanos, y entre estos y el espacio y el tiempo en que se desarrollan.

Todos los artículos recogidos en las páginas siguientes subrayan esta aptitud del medio para alojar voces opuestas al statu quo y en favor de la sostenibilidad medioambiental, que, además, al contrario que lo que emiten los altavoces del sistema, no se contrapone a la prosperidad económica, sino que va de la mano del reparto justo de la riqueza y, por tanto, de la conciencia de clase. Asimismo, resulta interesante observar cómo este tipo de historietas demuestran que el cómic es un medio óptimo para desafiar las convenciones del pacto ficcional al proponer un juego de espejos que lo hermanan con el reportaje periodístico o el ensayo.

El monográfico, entremos en la concreción, se compone de cinco artículos, a los que se añade una historieta de tres páginas que sirve de pórtico y, en cierta manera, de marco programático a través de su carácter disidente. Así, la autora Nacha Vollenweider inicia el recorrido del dosier con «De Río Cuarto a Córdoba», páginas en las que trata sobre la problemática del agronegocio, la agroindustria y sus consecuencias en la provincia de Córdoba (Argentina). En esta zona se conserva únicamente el 3\% de su bosque nativo, en buena medida por la mala praxis de grandes monopolios como Bayer, BASF o Monsanto, responsables de la creación de un sistema de dependencia al modificar las semillas genéticamente para que únicamente funcionen con sus productos. 
Jorge Catalá-Carrasco (Newcastle University) y Christine M. Martínez (New York University) proponen en «La imaginación ecológica en el cómic español» una suerte de estado de la cuestión en torno a la variedad de cómics que se preocupan por temas ecológicos para establecer alianzas con movimientos ecologistas y, en consecuencia, desarrollar una crítica ecológica del capitalismo contemporáneo y la cultura de consumo. Por tanto, bien podría decirse que este artículo, mediante la remisión al cómic en tanto que medio comunicativo sumamente adecuado para difundir la concienciación ecológica como freno a las estructuras de poder, se antoja de consulta utilísima para investigadores que quieran obtener pistas en torno a la materia de estudio, esto es, como una hoja de ruta clarividente.

Juan Francisco Álvarez-Herrero (Universitat d'Alacant) es autor de «Mortadelo y Filemón y Superlópez contra el cambio climático. Cuando el cómic español esconde algo más que aventura y diversión», una exploración de la temática ecologista en la historieta clásica de humor española. En este caso, cabe aplaudir que se posen los ojos en obras de enorme calado popular puesto que, a menudo, cuando se trata de temáticas alternativas al poder, se suele prestar más atención a los ítems propiamente de autor y se dejan de lado los objetos mainstream, que, a su manera, ni que sea sin una voluntad estrictamente disidente, pueden resultar igualmente sugestivos. En este sentido, Álvarez-Herrero, pese a loar las buenas intenciones, remarca que el tratamiento tiende a una cierta superficialidad que no permite concienciar al lector de manera efectiva. Ello no obsta, en todo caso, para que su lectura pueda resultar productiva si, por ejemplo, se les sometiera a un planteamiento didáctico con el docente como mediador.

"Ante la cementización de la vida: ;Vamos a la playa!», a cargo de Azul Blaseotto (Universidad del Museo Social Argentino), es una interesante aportación de corte ensayístico en que se analiza un cómic de autoría propia para reflexionar, en tiempos de cambio climático y crisis social, ecológica y ambiental, sobre las capacidades de la novela gráfica para retratar el paraíso perdido al borde del mar ante el avance indiscriminado y suicida del desarrollo inmobiliario. Sin duda, la destrucción del paisaje en favor de la especulación urbanística es uno de los mayores dramas a los que nos enfrentamos como sociedad, hasta el punto de que casi se ha convertido en un terror tremendamente cotidiano.

En «Experiencias gráficas de Chantal Montellier y Ana Penyas para la concienciación medioambiental. La autobiografía subterránea» de Tatiana Blanco-Cordón (Universidad de Granada), se pretende dilucidar qué recursos iconotextuales resultan significativos para la transmisión del relato ecocrítico. Para ello, Blanco-Cordón toma como objetos de análisis las novelas gráficas Tchernobyl mon amour (Chantal Montellier, 2006) y Todo bajo el sol (Ana Penyas, 2021), dos excelentes ejemplos de cómics recientes que abordan lo medioambiental y que, pese a hacerlo a partir de temáticas y contextos diferentes, comparten mecanismos narrativos merecedores de un análisis sosegado, sobre todo por lo que se refiere a la intersección de lenguajes mediante la técnica del collage.

Finalmente, Laura Caraballo (Université Clermont Auvergne) propone en «La historieta como vehículo de conocimiento en riesgos naturales. Algues vertes: l'histoire interdite» una 
compleja reflexión en torno a la especificidad de la historieta a la hora de transmitir conocimientos - por mor de las elecciones cromáticas, formales y los diferentes modos de representación y de puesta en página- y, yendo aún más allá, quizá para transformar la sociedad. Para ello, Caraballo se se detiene en una obra de la periodista Inès Van Léraud en colaboración con el dibujante Pierre Hove y la colorista Mathilda, una novela gráfica que presenta una larga investigación periodística sobre las mareas verdes en la región de Bretaña y que se caracteriza, entre otras cosas, por aportar un bagaje documental notable - estadísticas, opiniones, testimonios, explicitación de operaciones mediáticas y lobbies, etc.

Una vez concluida la panorámica a manera de captatio, los coordinadores nos despedimos con la esperanza de que este dosier se convierta en un modelo de consulta a tener en cuenta en la conformación de una masa crítica en torno a la temática medioambiental en el cómic. Pese a ser una materia en ebullición, el corpus bibliográfico se encuentra aún en un proceso relativamente primerizo - al menos, en cuanto a monografías-, por lo que cualquier esfuerzo, siempre que se base en el rigor metodológico, es bienvenido. Ojalá, pues, que este número 17 de Cuadernos de Cómic se alinee con otros referentes de la ecocrítica como EcoComix: Essays on the Environment in Comics and Graphic Novels, coordinado por Sidney I. Dobrin (2020) y enraizado en la tradición angloamericana, o el especial «What Grows in the Gutter? Eco-Comics» en el volumen 7 de la revista alemana Closure, bajo la coordinación de Cord-Christian Casper (2020).

Finalmente, no queremos bajar el telón sin dar las gracias a los articulistas por su buen hacer y, por descontado, también a los responsables de la revista por acoger la propuesta. Esto no es el final... 